\title{
Should CD4 Levels be Monitored in a Patient on Temozolomide?
}

\author{
Sriram Gonakoti, Luis Parra-Rodriguez \\ John H Stroger Jr Hospital of Cook County, Chicago, Illinois, USA
}

Doi: 10.12890/2021_002276 - European Journal of Case Reports in Internal Medicine - ๑ EFIM 2021

Received: 08/01/2021

Accepted: 25/02/2021

Published: 11/03/2021

How to cite this article: Gonakoti S, Parra-Rodriguez L. Should CD4 levels be monitored in a patient on temozolomide? EJCRIM 2021;8: doi:10.12890/2021_002276

Conflicts of Interests: The Authors declare that there are no competing interests.

This article is licensed under a Commons Attribution Non-Commercial 4.0 License

\section{ABSTRACT}

Introduction: Temozolomide is an alkylating agent, indicated in the treatment of refractory anaplastic astrocytoma and newly diagnosed glioblastoma. We describe a case of Salmonella typhimurium bacteraemia associated with septic arthritis in the setting of concurrent temozolomide use.

Case: A 67-year-old woman presented with acute onset of bilateral knee pain and swelling. She had a medical history of glioblastoma multiforme treated with temozolomide. Synovial fluid analysis and blood cultures revealed S. typhimurium, confirming a diagnosis of $S$. typhimurium bacteraemia associated with septic arthritis.

Conclusion: We conclude that chemotherapy with temozolomide and corticosteroid use will increase an individual's susceptibility to a wide variety of opportunistic infections akin to HIV-associated acquired immunodeficiency syndrome (AIDS). Furthermore, we hypothesize a possible benefit of monitoring CD4 levels and prophylaxis against opportunistic infections (based on the CD4 levels) in individuals receiving temozolomide-based chemotherapy, similar to HIV-AIDS.

\section{LEARNING POINTS}

- Chemotherapy with temozolomide and corticosteroid use will increase an individual's susceptibility to a wide variety of opportunistic infections akin to HIV-associated acquired immunodeficiency syndrome (AIDS).

- There may be a benefit in monitoring CD4 levels in patients who are receiving temozolomide.

- Given selective CD4 lymphopenia while on temozolomide, there may be a possible benefit of prophylaxis against opportunistic infections in individuals based on their CD4 levels, similar to HIV-AIDS (current guidelines recommend considering Pneumocystis jirovecii pneumonia prophylaxis for patients receiving concomitant radiotherapy and temozolomide, and high-dose steroids).

\section{KEYWORDS}

Temozolomide, non-typhoidal salmonellae, septic arthritis

\section{CASE DESCRIPTION}

Background

Temozolomide is an alkylating agent, indicated in the treatment of refractory anaplastic astrocytoma and newly diagnosed glioblastoma. We describe a case of Salmonella typhimurium bacteraemia associated with septic arthritis in the setting of concurrent temozolomide use. We discuss the current guidelines regarding antibiotic prophylaxis for patients on temozolomide therapy. 


\section{Case Presentation}

A 67-year-old woman with a left parietal glioblastoma multiforme (GBM) (WHO grade IV), heart failure with reduced ejection fraction (HFrEF), and mild bilateral knee osteoarthritis, presented to the hospital with progressive bilateral knee pain and swelling for 8 days. The pain was worsened by movements of the knees, with limitation of ambulation. There was no history of preceding trauma. She also described a 4-day history of chills and watery diarrhoea before symptom onset, which had resolved before admission.

She had been diagnosed with a left parietal GBM, WHO grade IV, diagnosed after a left parietal craniotomy for excisional biopsy of a brain mass, 7 months before the current presentation. She was currently on temozolomide (120 mg daily for 5 days every 4 weeks) and dexamethasone (2 mg every 12 hours). The patient was prescribed trimethoprim-sulfamethoxazole for Pneumocystis jirovecii pneumonia (PJP) prophylaxis upon commencement of chemotherapy. However, she was non-compliant with this medication.

The patient denied a history of tobacco consumption, illicit drug use or alcohol abuse. She did not have contact with pets or animals. She was born in El Salvador and had migrated to the United States over 20 years previously. The patient denied recent travel.

On physical examination, her blood pressure was $114 / 61 \mathrm{mmHg}$, heart rate was 82 beats per minute, respiratory rate was 14 breaths per minute, pulse oximetry was $95 \%$ on room air, and temperature was $36.3^{\circ} \mathrm{C}$. The musculoskeletal examination revealed a large left knee effusion with overlying erythema and increased warmth and tenderness. There was a small right knee effusion as well. Active and passive range of motion was painful and limited in both knees. The rest of the examination was otherwise normal.

Laboratory test results on admission are given in Table 1. The patient presented with leukopenia, lymphopenia, mild normocytic normochromic anaemia, and markedly elevated ESR and CRP. Note that CD4 levels were not checked during her admission. Plain radiography of the left knee demonstrated a left suprapatellar effusion with soft tissue swelling and mild multicompartment degenerative changes. Plain radiography of the right knee demonstrated mild multicompartment degenerative changes.

\begin{tabular}{|l|l|l|}
\hline Test & Result & Normal range \\
\hline Haemoglobin & $11.4 \mathrm{~g} / \mathrm{dl}$ & $11.7-14.9 \mathrm{~g} / \mathrm{dl}$ \\
\hline Platelet count & $161,000 \mathrm{cells} / \mathrm{\mu l}$ & $161,000-369,000 \mathrm{cells} / \mu \mathrm{l}$ \\
\hline $\begin{array}{l}\text { White blood cell } \\
\text { (WBC) count }\end{array}$ & $3,600 \mathrm{cells} / \mu \mathrm{l}$ & $4,400-10,600 \mathrm{cells} / \mu \mathrm{l}$ \\
\hline Absolute neutrophil count & $2,500 \mathrm{cells} / \mu \mathrm{l}$ & $2,200-6,900 \mathrm{cells} / \mu \mathrm{l}$ \\
\hline $\begin{array}{l}\text { Absolute lymphocyte count } \\
\text { Erythrocyte sedimentation } \\
\text { rate (ESR) }\end{array}$ & $1000 \mathrm{~mm} / \mathrm{hr}$ & $1,200-3,400 \mathrm{cell} / \mathrm{s} / \mu \mathrm{l}$ \\
\hline C-reactive protein (CRP) & $40 \mathrm{mg} / \mathrm{dl}$ & $0-39 \mathrm{~mm} / \mathrm{hr}$ \\
\hline
\end{tabular}

The patient was admitted to the hospital and underwent bilateral knee arthrocentesis. The results of synovial fluid analysis are given in Table 2. The synovial fluid culture was positive for S. typhimurium. The isolate was sensitive to ampicillin, ciprofloxacin and trimethoprimsulfamethoxazole. Blood cultures obtained on admission were positive for gram-negative rods, which eventually speciated to S. typhimurium, confirming a diagnosis of disseminated salmonellosis. There was no evidence of vegetations on a transthoracic echocardiogram.

Magnetic resonance imaging (MRI) of bilateral knees confirmed the presence of bilateral knee effusions (Fig. 1) without evidence of osteomyelitis or loculated abscesses.

The patient was treated with intravenous ceftriaxone $2 \mathrm{~g}$ daily and underwent bilateral knee debridement and irrigation. Intraoperatively, synovial fluid and synovium from both knees were sent for culture, which was positive for $S$. typhimurium. She required multiple irrigations and debridement of both knees, with eventual corticotomy of bilateral distal femurs. The patient improved progressively and was discharged home with instructions to complete a 4-week course of treatment with oral trimethoprim-sulfamethoxazole. 


\begin{tabular}{|c|c|c|c|c|c|}
\hline $\begin{array}{c}\text { Arthrocentesis } \\
\text { no. }\end{array}$ & Site & Appearance & 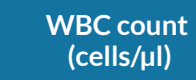 & $\begin{array}{c}\text { Differential } \\
\text { neutrophil count (\%) }\end{array}$ & $\begin{array}{l}\text { RBC count } \\
\text { (cells/ } / \mu \mathrm{I})\end{array}$ \\
\hline 1 & Left knee & Cloudy, orange & 13,000 & $94 \%$ & 9000 \\
\hline 2 & Left knee & Mucoid, orange & 85,000 & $95 \%$ & 32,000 \\
\hline 3 & Left knee & Mucoid, bloody & 290,000 & $80 \%$ & 289,000 \\
\hline
\end{tabular}

Table 2. Synovial fluid analysis

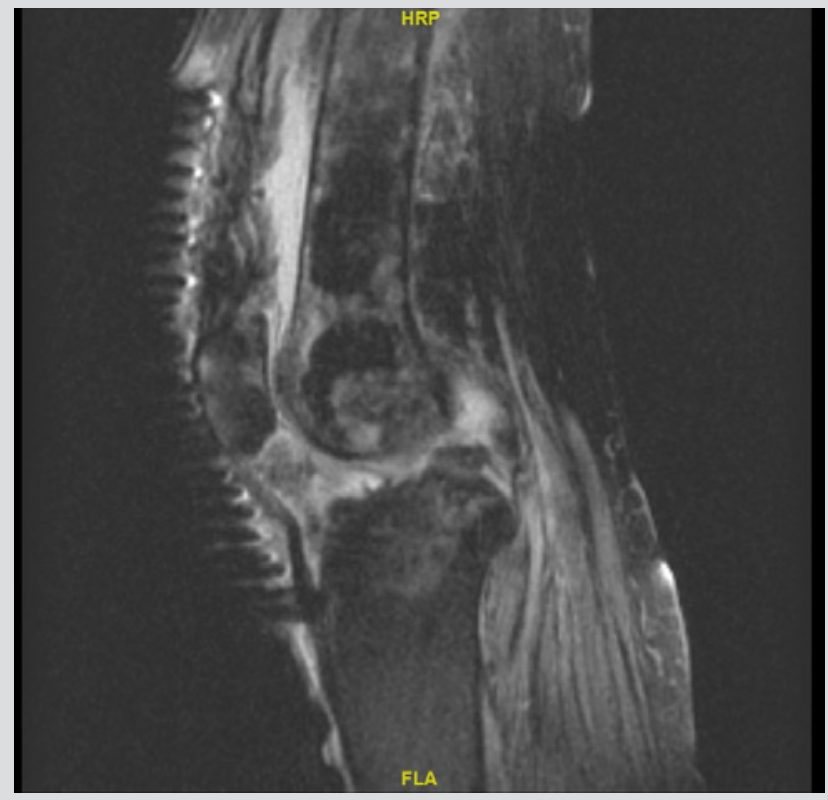

Figure 1. T2-weighted MRI of the right knee demonstrating moderate effusion

\section{DISCUSSION}

Temozolomide is an alkylating agent, indicated in the treatment of refractory anaplastic astrocytoma and newly diagnosed glioblastoma. One of the adverse effects is bone marrow suppression, which results in anaemia, thrombocytopenia, neutropenia and lymphocytopenia. In particular, selective CD4 lymphopenia has been described with extended use of temozolomide ${ }^{[1]}$. This is associated with an increased incidence of opportunistic infections such as PJP, mucocutaneous candidiasis, cryptococcosis, aspergillosis, blastomycosis, histoplasmosis, CMV, herpes zoster, herpes simplex and hepatitis $B^{[2,3]}$. Temozolomide use has been associated with disseminated salmonellosis with septic arthritis ${ }^{[4]}$. A reduction in CD4+ T-lymphocytes due to temozolomide could predispose patients to an increased incidence of disseminated salmonellosis. The presence of malignancy itself and the use of corticosteroids further predisposed our patient to salmonella bacteraemia. Current guidelines ${ }^{[5]}$ recommend considering PJP prophylaxis for patients receiving concomitant radiotherapy and temozolomide, and highdose steroids. PJP prophylaxis with trimethoprim-sulfamethoxazole could provide antibiotic coverage against gram-negative organisms such as Salmonella.

Given selective CD4+ T-lymphopenia with temozolomide use, there may be a possible benefit in monitoring the CD4 counts. Also, given the risk of opportunistic infections, akin to human immunodeficiency virus (HIV) associated with acquired immunodeficiency syndrome (AIDS), clinicians could consider antibiotic prophylaxis based on CD4 counts. Note that CD4 counts were not monitored for our patient (we were unaware of the rationale during her inpatient stay). There is a possibility that if they had been monitored, the importance of antibiotic prophylaxis would have been reinforced to our patient.

In most centres in developed countries, a CD4 count can be obtained within a few days. However, it may take longer in resource-poor settings. In these settings, monitoring the total lymphocyte count may play a role as a possible alternative. Although multiple studies have assessed the use of total lymphocyte count as a predictor for CD4 counts, there have been conflicting results. A thorough assessment of other immunosuppressive risk factors is essential. 


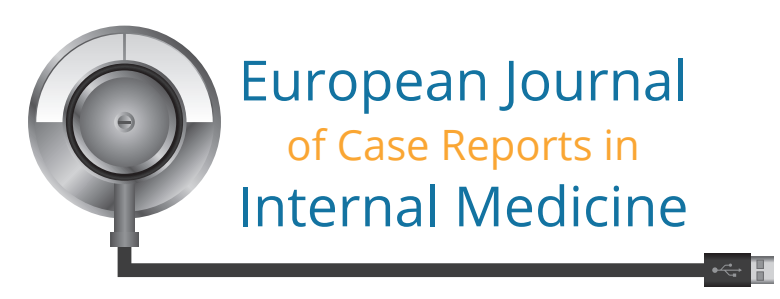

In conclusion, chemotherapy with temozolomide and corticosteroid use will increase an individual's susceptibility to a wide variety of opportunistic infections, similar to HIV-AIDS. Evaluation for non-typhoidal Salmonella infection must be considered as a part of the infectious work-up in a patient with a history of current temozolomide use. Furthermore, we hypothesize a possible benefit of monitoring CD4 levels (or total lymphocyte count) and prophylaxis against opportunistic infections (based on the CD4 levels/total lymphocyte count) in individuals receiving temozolomide-based chemotherapy, similar to HIV-AIDS.

\section{REFERENCES}

1. Su YB, Sohn S, Krown SE, Livingston PO, Wolchok JD, Quinn C, et al. Selective CD4+ lymphopenia in melanoma patients treated with temozolomide: a toxicity with therapeutic implications. J Clin Oncol 2004;22(4):610-616 [erratum in J Clin Oncol 2004;22(10):2038].

2. Stepanovic A, Nikitovic M. Severe hematologic temozolomide-related toxicity and life-threatening infections. J BUON 2018;23(1):7-13.

3. Kizilarslanoglu MC, Aksoy S, Yildirim NO, Ararat E, Sahin I, Altundag K. Temozolomide-related infections: review of the literature. J BUON 2011;16(3):547-550.

4. Georgescu G, Isola IM, Youssef S, Adachi J, Rolston K. Disseminated salmonellosis in a patient treated with temozolomide. J Infect 2008;57(5):414-415.

5. National Comprehensive Cancer Network (NCCN). Clinical Practice Guidelines in Oncology. Prevention and treatment of cancer-related infections. Version 2.2020. http:// www.nccn.org 